\title{
Opiate agonist treatment to improve health of individuals with opioid use disorder in Lebanon
}

\author{
Ali Ghaddar ${ }^{1,2^{*}}$ (D), Zeinab Abbas ${ }^{3,4}$ and Ramzi Haddad ${ }^{5,6}$
}

\begin{abstract}
Background: Opioid agonist therapy has been widely used to reduce harms among individuals with opioid use disorder but its effectiveness has not been evaluated in the Middle East North African (MENA) region. This study aims to evaluate the effectiveness of a program using opioid agonist therapy in combination with psychosocial support on improving psychological and social well-being, reducing arrest, and reducing risky behavior in individuals with opioid use disorder in Lebanon.

Methods: A one-group pre-test post-test design study was performed at SKOUN Lebanese Addiction Centre between January 2013 and December 2014. Eighty-six out of 181 patients agreed to participate and completed the 3-month assessment and 38 concluded the 12-month assessment. Psychological (depression and anxiety, quality of life), substance dependence/abuse, behavioral (injecting behavior, sharing needles and paraphernalia), and social outcomes were evaluated at baseline, 3, and 12 months post-treatment.

Results: Remarkable statistical significance improvements were observed 3 months after treatment in most outcome variables including quality of life, anxiety, substance dependence, overdose, employment, and injecting behavior. Improvements were sustained 12 months after treatment.

Conclusion: Results support expanding the access to opioid agonist therapy in other MENA countries to treat substance dependence and reduce harms among individuals with opioid use disorder.
\end{abstract}

Keywords: Buprenorphine, Harm reduction, Opiate-related disorders, Lebanon

\section{Background}

Opioid agonist treatment (OAT) has become the main treatment approach for people with opioid dependency and a fundamental component of the evidence-based harm reduction approach to HIV prevention in many developed countries [1]. Its implementation has resulted in a marked reduction in HIV-related risky behavior including injecting needles and sharing syringes leading to a decline in HIV transmission, HIV incidence, and mortality associated with unsafe injection $[2,3]$ and in preventing the spread of hepatitis $C$ virus among injecting drug users (IDUs) [4]. OAT has been increasingly used in order to decrease the health, economic, and

\footnotetext{
* Correspondence: ali.ghaddar@liu.edu.lb

${ }^{1}$ Department of Biomedical Sciences, Lebanese International University, Beirut, Lebanon

${ }^{2}$ Observatory of Public Policies and Health, Beirut, Lebanon

Full list of author information is available at the end of the article
}

social consequences of substance abuse and to improve quality of life (QoL) of opioid-dependent users [5-8].

OAT involves the provision of opioid agonist such as methadone and buprenorphine. The choice of the drug and of the modality of its provision (observed vs. takehome) influences treatment outcomes, yet there is little consensus about the most effective treatment regime [9]. A synthesis of evidence obtained from placebo-controlled trials revealed that buprenorphine is effective in suppressing illicit opioid use [9] and in (at higher doses) retaining patients in treatment although to a lesser extent than methadone $[10,11]$. On the other hand, buprenorphine has longer duration and limited withdrawal syndrome, and patients who receive buprenorphine were more likely to test negative for opioid use compared to those receiving methadone [12]. It also has a safer profile over methadone in terms of reducing mortality [11] and reducing 
diversion-related death [13]. In general, international guidelines emphasize direct observational induction of buprenorphine followed by multiple in-clinic visits [14]. However, with less strict supervision, unobserved takehome induction supposes fewer logistic barriers and thus results in better treatment outcomes including prolonged abstinence from opioids and reduced drug use $[15,16]$.

Despite its proven effectiveness and affordability, OAT is still unavailable in many low- and middle-income countries where it is desperately needed [1]. In such countries, evidence from a WHO collaborative study suggested that OAT reduced illegal opioid use, HIV-associated risk behaviors, and criminality, and substantially improved physical and mental health among opioid-dependent patients [17]. OAT also resulted in substantial improvements in QoL and in several domains of Addiction Severity Index including drug use, psychological well-being, criminality, and family relations [18]. OAT also demonstrated positive impact on QoL and physical, psychological, and social well-being among opioid users in Malaysia, Lithuania [19, 20], and Ukraine [21] and on reducing injecting behavior, criminality, HIV infections, and improving QoL in China [22-24]. Similarly, in Taiwan, OAT showed significant improvements in health-related QoL, psychological, and social wellbeing, and HIV-related risky behavior [25]. Indeed, there is increasing evidence suggesting that OAT could attain consistent outcomes in a culturally diverse range of settings in both low- and middle-income and high-income countries. In another study, OAT significantly reduced arrest incidences, risky behavior, and improved QoL [26]. Likewise, other studies encouraged to scale up OAT in low- and middle-income countries to save lives and resources [2]. This issue is of particular importance as the epidemic of dependence on prescription opioids is predicted to spread to low- and middle-income countries [27].

So far, OAT has been provided in at least five Middle East and North African (MENA) countries. Morocco developed a national plan that enabled the introduction of methadone substation treatment pilot programs in 2010. Bahrain, United Arab Emirates, and Palestine have also recently started providing OAT. Iran adopted OAT in its national policy and is the pioneer and leader in outpatient programs with estimated 4275 dispensing centers. In MENA countries, OAT program outcomes have been evaluated only in Iran where evidence support its effectiveness in reducing drug use, injection behavior, and syringe sharing and in improving health, QoL [28], and social well-being among IDUs [29].

In 2011, Lebanon adopted an OAT take-home buprenorphine pilot program only provided by authorized psychiatrists working within pre-registered treatment settings. One particularity of the treatment modality in Lebanon is the provision of psychosocial support as a basic component of the treatment. After being assessed for eligibility to treatment (diagnosis with opioid use disorder according to DSM5 criteria), patients are followed on weekly basis by a multidisciplinary team consisting of a psychiatrist, psychologist, social worker, and registered nurse. During follow-up, patients are monitored through regular urine tests for opiates, buprenorphine, and other drugs and are assessed for possible misuse, diversion, stability, and response to treatment. Furthermore, it is worth to mention that in Lebanon, a national study conducted in 2010 showed that heroin use accounted for $50 \%$ of patients treated for addiction and described a high rate of relapse among heroin users. It also revealed a high rate of arrest related to heroin use, with half of the treated patients having been already arrested at least once by the police [30]. Another particularity of OAT in Lebanon is that it could be a way out of arrest related to drug use, as drug use is criminalized in Lebanon except for users registered in treatment programs.

Almost 6 years has passed since the program initiation in Lebanon in 2011, yet, there exist no published reports about its effectiveness. Furthermore, the effectiveness of an OAT approach combining the component of psychosocial support provided by multidisciplinary team has not been well explored in previous research. The current study aims to evaluate the effectiveness of the pilot OAT program in Lebanon implemented by multidisciplinary teams on treating substance use disorders, improving the mental health and social functioning, and in reducing risky behavior among individuals with opioid use disorder. It also aims to explore the program outcome on reducing arrest related to drug use among registered users.

\section{Methods}

\section{Assessment}

The Lebanese Public Health Ministry (MOPH) implemented buprenorphine for treating opioid use disorder as evidence documents its feasibility, comparable safety profile, and fewer logistic barriers than observed induction [14]. The national guidelines specify that patients receive weekly take-home buprenorphine prescribed by authorized psychiatrists working within pre-registered treatment settings. Eligible patients diagnosed with opioid use disorder according to DSM5 or ICD10 (WHO) criteria are followed up on weekly basis by multidisciplinary teams consisting of a psychiatrist, psychologist, social worker, and registered nurse. Patients are monitored through regular urine tests for opioids and buprenorphine and are assessed for possible misuse, stability, and response to treatment during regular visits to treatment center.

Upon admission, participants completed a selfadministered questionnaire that included variables on socio-demographic characteristics, medical history, and substance use. Participants were interviewed by trained psychologists that were part of the treatment team and 
were trained for the purpose of the project. For each patient, questionnaires were filled systematically at three time intervals: upon admission (baseline), 3, and 12 months post-treatment.

The research protocol was approved by the institutional review boards at SKOUN and at the Lebanese International University. A one-group pre-test post-test design was adopted in order to measure the effect of OAT by examining the difference in the outcomes at baseline, 3 , and 12 months post-treatment.

\section{Participants}

SKOUN Lebanese Addiction Center is the first and largest outpatient community-based treatment center to implement OAT in Lebanon and currently enrolls around $40 \%$ of the OAT patients in Lebanon. Participants in the study were opioid-dependent patients who sought treatment at SKOUN. During the study period (January 2013-December 2014), patients diagnosed with opioid use disorder according to the Diagnostic and Statistical Manual of Mental Disorders (DSM5) who were prescribed OAT at SKOUN were approached for participation (181 male patients). Women were not included as there is a very low prevalence of women seeking treatment, especially for heroin dependence. Participants were clearly explained that refusal to participate will not affect their treatment process. Eighty-six patients agreed to participate and concluded the 3-month follow-up assessment, out of which 38 concluded the 12-month follow-up assessment. Participants were briefed by the team members about the objectives of the research and signed a written informed consent prior to participation. Patients were explained that refusal to participate will not affect positively or negatively their treatment. Ethical approval was obtained from the Institutional Review Board of the Lebanese International University.

\section{Measures}

Participants were asked about their age, educational level, average household income, marital status, employment criminal activity, number of arrests, and number of days in prison. Several participants had along with heroin dependence other substance dependence (cocaine and cannabis). We monitored the outcome of OAT on reducing heroin dependence along with cocaine and cannabis dependence. Substance use disorder (i.e., heroin, cocaine, and cannabis) was assessed according to DSM5 criteria (American Psychiatric Association, 2000).

Self-reports concerning participants' general health in the last 2 weeks were measured using EUROHIS QoL Scale (WHO QOL-8) validated questionnaire [31]. The scales were composed of eight questions with Likerttype scale with five response options $(1=$ very poor, $5=$ very good). The utilized scales were translated and blindly back translated in Arabic by two separate professional translators. An expert panel helped solve discrepancies between both translations and the final version was used.

The Hospital Anxiety and Depression Scale (HADS) was used to assess psychological well-being [32]. Each of anxiety and depression was measured by seven items with Likert-type scale with four response options $(0=$ not at all, $3=$ most of the times). Anxiety was assessed by asking whether participants experienced tense or frightened feelings, worrying thoughts, or whether they felt restless. Depression was assessed through questions such as "I have lost interest in my appearance." The score of each dimension is established by the summation of the individual questions. Cronbach's alpha reliability coefficients of the scales in the sample were $0.77,0.84$, and 0.81 for QoL, anxiety, and depression, respectively.

Injecting behavior was measured through a scale from 1 to $4(1=$ during last 3 months and $4=$ never $)$ and sharing needles was measured through a scale from 1 to $6(1=$ more than 10 times and $6=$ never $)$ based on a questionnaire that measures risk for transmission of blood-borne viruses [33]. Overdose was measured through a simple question about overdose during last 3 months.

\section{Statistical analysis}

We displayed in the results only the pretreatment variables that explained at least $1 \%$ of the variance in our health outcomes (Table 1). The other pre-treatment variables that could not account for variance in the outcome variables (eta-square less than 0.01 ) are not mentioned in the table. Shapiro-Wilk test was used to check the normality of distribution of the outcome variables. For continuous variables that showed normal distribution (QoL, anxiety, and depression), within-group changes were analyzed using paired, one-tailed $t$ test (for the 3-month assessment) and one-way repeated measure ANOVA (for the 12-month assessment). For the other variables that were not normally distributed, changes in nominal categorical variables (previous arrest, work) were analyzed using the McNemar non-parametric tests with effect sizes calculated in accordance with Cohen's $d$. Differences in ordinal variables (injecting behavior and sharing needles) were analyzed by $z$-Wilcoxon rank non-parametric tests with calculating $r$-correlation coefficient for effect size. $\alpha$-level of $5 \%$ was considered statistically significant.

\section{Results}

\section{Sample characteristics}

The baseline characteristics of participants who completed the 3- and the 12-month assessment are displayed in Table 1. Participants age ranged between 18 and 
Table 1 Pre-treatment individual characteristics of participants who completed the 3-month assessment $(n=86)$ and the 12-month assessment $(n=38)$

\begin{tabular}{lll}
\hline & 3 months & 12 months \\
\hline Age, years & $n=86$ & $n=38$ \\
$18-25$ & $32(37.2 \%)$ & $15(39.5 \%)$ \\
$26-30$ & $25(29.1 \%)$ & $11(28.9 \%)$ \\
$31-35$ & $20(23.3 \%)$ & $9(23.7 \%)$ \\
$36+$ & $9(10.5 \%)$ & $3(7.9 \%)$ \\
Educational level & $n=84$ & $n=37$ \\
Illiterate & $19(22.6 \%)$ & $5(13.5 \%)$ \\
School & $44(52.4 \%)$ & $19(51.4 \%)$ \\
University & $21(25.0 \%)$ & $13(35.1 \%)$ \\
Average household income, $\$$ & $n=77$ & $n=34$ \\
Low (< 1000) & $43(50.0 \%)$ & $20(52.6 \%)$ \\
Middle (1000-2500) & $25(29.1 \%)$ & $11(28.9 \%)$ \\
High (2500+) & $9(10.5 \%)$ & $3(7.9 \%)$ \\
Marital status & $n=86$ & $n=38$ \\
Never married & $60(69.8 \%)$ & $24(63.2 \%)$ \\
Ever married & $26(30.2 \%)$ & $14(36.8 \%)$ \\
Number of times arrested & $n=86$ & $n=38$ \\
None & $24(27.9 \%)$ & $10(26.3 \%)$ \\
1 & $20(23.3 \%)$ & $11(28.9 \%)$ \\
$\geq 2$ & $42(48.8 \%)$ & $17(44.7 \%)$ \\
Longest duration of detainment, days & $n=86$ & $n=38$ \\
0 & $23(26.7 \%)$ & $10(26.3 \%)$ \\
1-30 & $21(24.4 \%)$ & $12(31.6 \%)$ \\
Substance concern 1 month & $42(48.8 \%)$ & $16(42.1 \%)$ \\
Heroine & $n=86$ & $n=38$ \\
\hline & $76(89.4 \%)$ & $36(94.7 \%)$ \\
Cocaine & $10(11.6 \%)$ & $6(15.8 \%)$ \\
\hline
\end{tabular}

66 years with median $=28$ years $($ standard deviation $=7.61)$. Around $73 \%$ of participants had history of arrest. The number of previous arrests ranged between 0 and 18 times with median $=2$ times $($ standard deviation $=2.86$ ). Participants stayed in prison between 0 and 6330 days with median = 20 days (standard deviation $=716.27$ days). Around half of the participants had school education and had low average household income, and the majority were never married.

Results indicated that the treatment had positive effects on the quality of life, anxiety, and depression that were sustained over the two post-treatment time intervals. QoL scores significantly increased while anxiety scores significantly decreased 3 and 12 months after the treatment. Likewise, depression scores dropped after the treatment, although the difference in scores compared to baseline was statistically significant at the 3-month interval but lost significance at the 12-month interval. However, although reductions in anxiety scores were maintained 12 months after the treatment, the difference between baseline scores of anxiety lost statistical significance (Table 2).

The treatment had positive effects on reducing arrest and improving employment over time. McNemar's test determined that there was a statistically significant reduction in the proportion of persons arrested, at the two time intervals (3 and 12 months) post-treatment compared to baseline. However, the \% of employed patients increased 3 months after treatment (statistically significant difference) and 12 months after treatment (non-significant difference). Similarly, the McNemar test showed statistically significant reductions in the \% of patients who reported overdosing and the \% of patients who met heroin, cocaine, and cannabis dependence criteria, at both the 3 and 12 months' time intervals after the treatment (Table 3).

Results of the Wilcoxon $z$-non-parametric test indicated a slight, statistically non-significant reduction in injecting behavior scores at the 3-month interval $(z=-1.23$; $p$ value $=0.15$; effect size $r=0.09)$ and the 12-month intervals $(z=-0.56$; $p$ value $=0.21$; effect size $r=0.07)$. Similarly, the Wilcoxon $z$ test showed significant reduction in the score of sharing needles 3 months $(z=-1.67 ; p$ value $=0.001$; effect size $r=0.12$ ) and non-significant reduction in the scores 12 months after the treatment $(z=-0.22 ; p$ value $=0.82$; effect size $r=0.03)$. Fewer patients reported overdosing after 3 and 12 months compared to baseline. Significant reductions were noted in the $\%$ of patients who met opioid use disorder, cocaine use disorder, and cannabis use disorder 3 and 12 months after treatment.

\section{Discussion}

The primary outcome evaluation of OAT pilot program in Lebanon supports its implementation for treating substance use disorder and reducing harms among individuals with opioid use disorder. Remarkable statistically significant improvements were observed over time (3 and 12 months) among patients under buprenorphine in most outcome variables including QoL, anxiety, substance dependence, overdose, and employment. Congruent findings have been observed in low- and middle-income countries about the effectiveness of buprenorphine in improving mental health and in reducing risky behaviors [17].

The most impressive finding was improvement in employment, not something usually noted in evaluations performed in high-income countries. Statistically significant improvements in QoL were documented after treatment, in consistence with previous findings in lowand middle-income countries [18]. Contrary to findings obtained in other countries, the percentage of arrest 
Table 2 Changes in mean (s.d.) in outcomes 3 and 12 months post-treatment

\begin{tabular}{|c|c|c|c|c|c|c|c|c|c|}
\hline \multirow[b]{2}{*}{ Variable and group } & \multicolumn{4}{|c|}{ 3-month assessment $(n=75)$} & \multicolumn{5}{|c|}{ 12-month assessment $(n=36)$} \\
\hline & Baseline & Post 3 months & $p$ value & Effect size & Baseline & Post 3 months & Post 12 months & $p$ value & Effect size \\
\hline $\begin{array}{l}\text { EUROHIS Quality of Life } \\
\text { Scale (8-40) mean (s.d.) }\end{array}$ & $21.82(6.59)$ & $24.93(6.32)$ & $p \leq 0.001$ & Cohen's $d=0.37$ & $22.37(6.22)$ & $24.16(5.82)$ & $24.84(6.92)$ & $p=0.05$ & $\eta 2=0.14$ \\
\hline $\begin{array}{l}\text { Anxiety (HADS) (0-21) } \\
\text { mean (s.d.) }\end{array}$ & $9.62(4.09)$ & $7.29(4.55)$ & $p=0.001$ & Cohen's $d=0.34$ & $9.27(3.84)$ & $7.18(4.19)$ & $6.91(3.91)$ & $p=0.006$ & $\eta 2=0.24$ \\
\hline $\begin{array}{l}\text { Depression (HADS) } \\
(0-21) \text { mean (s.d.) }\end{array}$ & $8.97(3.56)$ & $7.66(3.69)$ & $p=0.03$ & Cohen's $d=0.21$ & $8.94(3.58)$ & $8.01(3.54)$ & $7.65(3.82)$ & $p=0.19$ & $\eta 2=0.17$ \\
\hline
\end{tabular}

HADS Hospital Anxiety Depression, s.d. standard deviation, $d$ Cohen's $d$ effect size, $\eta 2$ eta-squared effect size

Table 3 Changes in frequency and \% in outcomes 3 and 12 months post-treatment

\begin{tabular}{|c|c|c|}
\hline & $n(\%)$ & $p$ value \\
\hline \multicolumn{3}{|c|}{ Arrested last 3 months -3-month assessment $(n=86)$} \\
\hline Baseline & $62(72.1 \%)$ & \\
\hline 3 months & $7(8.1 \%)$ & 0.001 \\
\hline \multicolumn{3}{|c|}{ Arrested last 3 months-12-month assessment $(n=38)$} \\
\hline Baseline & $24(70.6 \%)$ & \\
\hline 12 months & $1(2.6 \%)$ & 0.001 \\
\hline \multicolumn{3}{|c|}{ Working -3-month assessment $(n=86)$} \\
\hline Baseline & $24(27.9 \%)$ & \\
\hline 3 months & $52(60.5 \%)$ & 0.02 \\
\hline \multicolumn{3}{|c|}{ Working-12-month assessment $(n=38)$} \\
\hline Baseline & $12(31.6 \%)$ & 0.07 \\
\hline 12 months & $30(78.9 \%)$ & \\
\hline \multicolumn{3}{|c|}{$N$ times overdose-3-month assessment $(n=86)$} \\
\hline Baseline & $12(14.0 \%)$ & \\
\hline 3 months & $3(3.5 \%)^{*}$ & 0.02 \\
\hline \multicolumn{3}{|c|}{$N$ times overdose-12-month assessment $(n=38)$} \\
\hline Baseline & $8(21.1 \%)$ & \\
\hline 12 months & $1(2.6 \%)$ & 0.03 \\
\hline \multicolumn{3}{|c|}{ Heroin use disorder-3-month assessment $(n=86)$} \\
\hline Baseline & $72(100 \%)$ & \\
\hline 3 months & $5(6.9 \%)$ & $\leq 0.001$ \\
\hline \multicolumn{3}{|c|}{ Heroin use disorder-12-month assessment $(n=38)$} \\
\hline Baseline & $38(100 \%)$ & \\
\hline 12 months & $1(2.6 \%)$ & $\leq 0.001$ \\
\hline \multicolumn{3}{|c|}{ Cocaine use disorder-3-month assessment $(n=86)$} \\
\hline Baseline & $13(18 \%)$ & \\
\hline 3 months & $1(1.3 \%)$ & $p=0.04$ \\
\hline \multicolumn{3}{|c|}{ Cocaine use disorder-12-month assessment $(n=38)$} \\
\hline Baseline & $6(15.8 \%)$ & \\
\hline 12 months & $1(2.6 \%)$ & $p=0.01$ \\
\hline \multicolumn{3}{|c|}{ Marijuana use disorder-3-month assessment $(n=86)$} \\
\hline Baseline & $18(25 \%)$ & \\
\hline 3 months & $6(9.7 \%)$ & $p=0.01$ \\
\hline \multicolumn{3}{|c|}{ Marijuana use disorder-12-month assessment $(n=38)$} \\
\hline Baseline & $8(21 \%)$ & \\
\hline 12 months & $3(7.8 \%)$ & 0.04 \\
\hline
\end{tabular}

among patients receiving OST significantly decreased after the treatment [34]. A possible explanation of this discrepancy in the results is that participants were arrested due to drug use, which is considered a criminal act in Lebanon. Registering in the OAT program is a way out of penalizing persons using drugs. This probably explains the high number of arrest reported before engaging in the program and the dramatic drop 3 months after treatment.

\section{Limitations}

The current study has some limitations related to the study population and design. The absence of a control group and of randomization poses limitations to internal validity related to the difficulty to exclude confounders. The sample size was not calculated a priori, was relatively small, and represented exclusively male heroindependent users. Finally, results are prone to information bias related to self-reporting as reported improvement could have been possibility overestimated. Actually, it could be argued that patients usually enter treatment at a time of crisis, when social functioning is poor and self-report is prone to overstate poor health. On the other hand, there is a selection bias related to non-response and loss to follow-up, as one could argue that those followed up are the ones who were successful and that only $47.5 \%$ concluded the 3-month assessment. A possible explanation for patient non-participation and dropout could be the transfer of patients to other centers or due to patients' own will to stop treatment. A more comprehensive evaluation is needed to monitor the benefits of the treatment on a longer term.

\section{Conclusion}

Results have important implications to guide policy makers in making informed decisions about treatment options of individuals with opioid use disorder. The challenges related to the difficult socio-political context that faced the implementation of OAT program in Lebanon since its launching in December 2011 should be taken into consideration [35]. Other MENA countries implementing OAT should also take into consideration the specificities of the treatment protocol adopted by the 
Lebanese pilot program and should address adopting alternative treatment provision protocols. For instance, in the Lebanese example, a positive evaluation of retention in the program was given to patients who tested negative in the weekly urine test. Following the latest evidence about using more frequent urine testing would allow for a more objective evaluation of the retention in the program and provide better outcomes on sustaining opioid-free urinalysis [36]. Further evaluations of the effectiveness of OAT countries are warranted to monitor the potential adverse effects associated with buprenorphine misuse in MENA [1,37]. The encouraging results of the evaluation of the first pilot OAT program in Lebanon support expanding the access to buprenorphine in Lebanon and other MENA countries in order to treat individuals with opioid use disorder.

\section{Abbreviations}

HIV: Human immunodeficiency virus; IDUs: Injecting drug users;

MENA: Middle East North Africa; MoH: Ministry of Public Health; OAT: Opioid agonist therapy; QoL: Quality of life

\section{Acknowledgements}

The authors acknowledge the support of the SKOUN Lebanese Addiction Center during the phases of study design and data collection. The authors also acknowledge Dr. Alaa Fawaz for revising the paper.

\section{Funding}

This study was funded by the National Council of Scientific Research (CNRS) - Lebanon.

\section{Availability of data and materials}

Data sharing is not applicable to this article as no datasets were generated or analyzed during the current study.

\section{Authors' contributions}

AG carried out the study design, evaluation, and data analysis and drafted the manuscript. ZA drafted parts of the manuscript and provided guidance as a consultant in the implementation of opioid substitution treatment programs. $\mathrm{RH}$ revised and adjusted the study design and coordinated the psychiatric follow-up and evaluation of the study participants. All authors read and approved the final manuscript.

\section{Ethics approval and consent to participate}

All procedures performed in studies involving human participants were in accordance with the ethical standards of the institutional and national research committee and with the 1964 Helsinki declaration and its later amendments or comparable ethical standards. Informed consent was obtained from all individual participants included in the study.

\section{Consent for publication}

Not applicable

\section{Competing interests}

The authors declare that they have no competing interests.

\section{Publisher's Note}

Springer Nature remains neutral with regard to jurisdictional claims in published maps and institutional affiliations.

\section{Author details}

'Department of Biomedical Sciences, Lebanese International University, Beirut, Lebanon. ${ }^{2}$ Observatory of Public Policies and Health, Beirut, Lebanon. ${ }^{3}$ School of Pharmacy, Lebanese International University, Beirut, Lebanon. ${ }^{4}$ Department of Narcotics, Ministry of Public Health, Beirut, Lebanon.
${ }^{5}$ Department of Psychiatry, Lebanese University, Beirut, Lebanon.

${ }^{6}$ Department of Psychiatry, Saint Joseph University, Beirut, Lebanon.

Received: 15 September 2017 Accepted: 29 November 2017

Published online: 08 December 2017

\section{References}

1. Mathers BM, Degenhardt L, Ali H, Wiessing L, Hickman M, Mattick RP, et al. HIV prevention, treatment, and care services for people who inject drugs: a systematic review of global, regional, and national coverage. Lancet. 2010; 375(9719):1014-28. doi: 10.1016/s0140-6736(10)60232-2.

2. Gowing LR, Hickman M, Degenhardt L. Mitigating the risk of HIV infection with opioid substitution treatment. Bull World Health Organ. 2013;91(2): 148-9. doi: 10.2471/blt.12.109553.

3. United Nations Office on Drugs and Crime (UNODC). World drug report. 2014. (United Nations Publications, Sales No. E. 14X17). https://www.unodc. org/documents/wdr2014/World_Drug_Report_2014_web.pdf

4. MacArthur G, van Velzen E, Palmateer N, Kimber J, Pharris A, Hope V, et al. Interventions to prevent HIV and hepatitis $C$ in people who inject drugs: a review of reviews to assess evidence of effectiveness. Int J Drug Policy. 2014;25(1):34-52. doi: 10.1016/j.drugpo.2013.07.001.

5. Brown L, Alterman A, Rutherford M, Cacciola J, Zaballero A. Addiction severity index scores of four racial/ethnic and gender groups of methadone maintenance patients. J Subst Abus. 1993;5(3):269-79.

6. Strain E, Stitzer M, Liebson I, Bigelow G. Buprenorphine versus methadone in the treatment of opioid dependence. J Clin Psychopharmacol. 1996;16(1):58-67.

7. Tracy E, Laudet A, Min M, Kim H, Brown S, Jun M, Singer L. Prospective patterns and correlates of quality of life among women in substance abuse treatment. Drug Alcohol Depend. 2012;124(3):242-9. doi: 10.1016/j. drugalcdep.2012.01.010

8. De Maeyer J, Vanderplasschen W, Broekaert E. Quality of life among opiatedependent individuals: a review of the literature. Int J Drug Policy. 2010; 21(5):364-80. doi: 10.1016/j.drugpo.2010.01.010.

9. Meader N. A comparison of methadone, buprenorphine and alpha2 adrenergic agonists for opioid detoxification: a mixed treatment comparison meta-analysis. Drug Alcohol Depend. 2010;108(1-2):110-4.

10. Mattick RP, Breen C, Kimber J, Davoli M. Buprenorphine maintenance versus placebo or methadone maintenance for opioid dependence. Cochrane Database Syst Rev. 2008; CD002207. doi: 10.1002/14651858.CD002207.pub3.

11. Connock M, Juarez-Garcia A, Jowett S, Frew E, Liu Z, Taylor R, et al. Methadone and buprenorphine for the management of opioid dependence: a systematic review and economic evaluation. Health Technol Assess. 2007;11(9):1-171.

12. West S, O'Neal K, Graham C. A meta-analysis comparing the effectiveness of buprenorphine and methadone. J Subst Abus. 2000;12(4):405-14.

13. Bell J, Butler B, Lawrance A, Batey R, Salmelainen P. Comparing overdose mortality associated with methadone and buprenorphine treatment. Drug Alcohol Depend. 2009;104(1-2):73-7. doi: 10.1016/j.drugalcdep.2009.03.020.

14. Lee J, Vocci F, Fiellin D. Unobserved "home" induction onto buprenorphine. J Addict Med. 2014;8(5):299-308. doi: 10.1097/ADM.0000000000000059.

15. Chawarski M, Mazlan M, Schottenfeld R. Behavioral drug and HIV risk reduction counseling $(\mathrm{BDRC})$ with abstinence-contingent take-home buprenorphine: a pilot randomized clinical trial. Drug Alcohol Depend. 2008; 94(1-3):281-4. https://doi.org/10.1016/j.drugalcdep.2007.11.008.

16. Cunningham C, Giovanniello A, Li X, Kunins H, Roose R, Sohler N. A comparison of buprenorphine induction strategies: patient-centered homebased inductions versus standard-of-care office-based inductions. J Subst Abus Treat. 2011;40(4):349-56. doi: 10.1016/j.jsat.2010.12.002.

17. Lawrinson P, Ali R, Buavirat A, Chiamwongpaet S, Dvoryak S, Habrat B, et al. Key findings from the WHO collaborative study on substitution therapy for opioid dependence and HIV/AIDS. Addiction. 2008;103(9):1484-92. doi: 10. 1111/j.1360-0443.2008.02249.x

18. Feelemyer J, Jarlais D, Arasteh $\mathrm{K}$, Phillips B, Hagan $\mathrm{H}$. Changes in quality of life (WHOQOL-BREF) and addiction severity index (ASI) among participants in opioid substitution treatment (OST) in low and middle income countries: an international systematic review. Drug Alcohol Depend. 2014;134:251-8.

19. Lua P, Talib N. A 12-month evaluation of health-related quality of life outcomes of methadone maintenance program in a rural Malaysian sample. Subst Use Misuse. 2012:47(10):1100-5. doi: 10.3109/10826084.2012.679840. 
20. Padaiga Z, Subata E, Vanagas G. Outpatient methadone maintenance treatment program. Quality of life and health of opioid-dependent persons in Lithuania. Medicina (Kaunas). 2007:43:235-41.

21. Morozova O, Dvoriak S, Pykalo I, Altice F. Primary healthcare-based integrated care with opioid agonist treatment: first experience from Ukraine. Drug Alcohol Depend. 2017;173:132-8. doi: 10.1016/j. drugalcdep.2016.12.025.

22. Pang L, Hao Y, Mi G, Wang C, Luo W, Rou K, et al. Effectiveness of first eight methadone maintenance treatment clinics in China. AIDS. 2007;21(Suppl 8): S103-7. doi: 10.1097/01.aids.0000304704.71917.64.

23. He Q, Wang X, Xia Y, Mandel J, Chen A, Zhao L, et al. New communitybased methadone maintenance treatment programs in Guangdong, China, and their impact on patient quality of life. Substance Use \& Misuse. 2010; 46(6):749-57.

24. Xiao L, Wu Z, Luo W, Wei X. Quality of life of outpatients in methadone maintenance treatment clinics. J Acquir Immune Defic Syndr. 2010:53:S116-20. doi: 10.1097/QAl.0b013e3181c7dfb5.

25. Wang P, Wu H, Yen C, Yeh Y, Chung K, Chang H, Yen C. Change in quality of life and its predictors in heroin users receiving methadone maintenance treatment in Taiwan: an 18-month follow-up study. Am J Drug and Alcohol Abuse. 2012;38(3):213-9. doi: 10.3109/00952990.2011.649222.

26. Armstrong G, Kermode M, Sharma C, Langkham B, Crofts N. Opioid substitution therapy in Manipur and Nagaland, North-East India: operational research in action. Harm Reduct J. 2010;7(1):29. doi: 10.1186/1477-7517-7-29.

27. Holmes D. Prescription drug addiction: the treatment challenge. Lancet. 2012;379(9810):17-8. doi: 10.1016/50140-6736(12)60007-5

28. Esmaeili $H$, Ziaddinni $H$, Nikravesh M, Baneshi M, Nakhaee N. Outcome evaluation of the opioid agonist maintenance treatment in Iran. Drug Alcohol Rev. 2014;33(2):186-93. doi: 10.1111/dar.12112.

29. Noori R, Narenjiha H, Aghabakhshi H, Habibi G, Khoshkrood Mansoori B. Methadone maintenance therapy outcomes in Iran. Subst Use Misuse. 2012; 47(7):767-73. doi: 10.3109/10826084.2010.517726

30. Karam EG, Ghandour LA, Maalouf WE, Yamout K, Salamoun MM. A rapid situation assessment (RSA) study of alcohol and drug use in Lebanon. J Med Liban. 2010;58:76-85.

31. Schmidt S, Muhlan H, Power M. The EUROHIS-QOL 8-item index: psychometric results of a cross-cultural field study. Eur J Pub Health. 2006; 11:420-8. doi: 10.1093/eurpub/cki155.

32. Zigmond AS, Snaith RP. The hospital anxiety and depression scale. Acta Psychiatr Scand. 1983;67(6):361-70.

33. Blood-Borne Virus and Sexual Health Screener. Network of Alcohol and other Drugs Agencies (NADA). Client Outcomes Management System (C. O. M. S). Australia; 2012. http://www.nada.org.au/whatwedo/nadabase-nmdscoms.

34. Harris E, Jacapraro J, Rastegar D. Criminal charges prior to and after initiation of office-based buprenorphine treatment. Subst Abuse Treat Prev Policy. 2012;7(1):10

35. El-Khoury J, Abbas Z, Nakhle P, Matar M. Implementing opioid substitution in Lebanon: inception and challenges. Int J Drug Policy. 2016;31:178-83. doi: 10.1016/j.drugpo.2016.02.015.

36. Chutuape M, Silverman K, Stitzer M. Effects of urine testing frequency on outcome in a methadone take-home contingency program. Drug Alcohol Depend. 2001;62(1):69-76. doi: 10.1016/\$0376-8716(00)00160-5.

37. Wright N, D'Agnone O, Krajci P, Littlewood R, Alho H, Reimer J, et al. Addressing misuse and diversion of opioid substitution medication: guidance based on systematic evidence review and real-world experience. J Public Health. 2015;38(3):e368-74. doi: 10.1093/pubmed/fdv150.

\section{Submit your next manuscript to BioMed Central and we will help you at every step:}

- We accept pre-submission inquiries

- Our selector tool helps you to find the most relevant journal

- We provide round the clock customer support

- Convenient online submission

- Thorough peer review

- Inclusion in PubMed and all major indexing services

- Maximum visibility for your research

Submit your manuscript at www.biomedcentral.com/submit
Biomed Central 